\title{
Transcriptome analysis of responses to bluetongue virus infection in Aedes albopictus cells
}

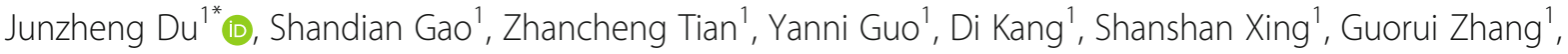 \\ Guangyuan Liu', Jianxun Luo ${ }^{1}$, Huiyun Chang ${ }^{1}$ and Hong Yin ${ }^{1,2}$
}

\begin{abstract}
Background: Bluetongue virus (BTV) causes a disease among wild and domesticated ruminants which is not contagious, but which is transmitted by biting midges of the Culicoides species. BTV can induce an intense cytopathic effect (CPE) in mammalian cells after infection, although Culicoides- or mosquito-derived cell cultures cause non-lytic infection with BTV without CPE. However, little is known about the transcriptome changes in Aedes albopictus cells infected with BTV.

Methods: Transcriptome sequencing was used to identify the expression pattern of mRNA transcripts in A. albopictus cells infected with BTV, given the absence of the Culicoides genome sequence. Bioinformatics analyses were performed to examine the biological functions of the differentially expressed genes. Subsequently, quantitative reverse transcriptionpolymerase chain reaction was utilized to validate the sequencing data.

Results: In total, 51,850,205 raw reads were generated from the BTV infection group and 51,852,293 from the control group. A total of 5769 unigenes were common to both groups; only 779 unigenes existed exclusively in the infection group and 607 in the control group. In total, 380 differentially expressed genes were identified, 362 of which were upregulated and 18 of which were down-regulated. Bioinformatics analyses revealed that the differentially expressed genes mainly participated in endocytosis, FoxO, MAPK, dorso-ventral axis formation, insulin resistance, Hippo, and JAK-STAT signaling pathways.

Conclusion: This study represents the first attempt to investigate transcriptome-wide dysregulation in A. albopictus cells infected with BTV. The understanding of BTV pathogenesis and virus-vector interaction will be improved by global transcriptome profiling.
\end{abstract}

Keywords: Aedes albopictus cells, Bluetongue virus, Transcriptome sequencing, Differentially expressed genes, Vector-virus interaction

\section{Background}

Bluetongue (BT) is a major non-contagious disease of ruminants transmitted by biting midges of the Culicoides genus. Bluetongue virus (BTV), the etiological agent of BT, is the type species of the Orbivirus genus, in the family Reoviridae [1-3]. Historically, the epidemic distribution was limited to tropical and warm temperate regions where

\footnotetext{
* Correspondence: dujunzheng@caas.cn

'State Key Laboratory of Veterinary Etiological Biology, Lanzhou Veterinary Research Institute, Chinese Academy of Agricultural Sciences, Xujiaping 1, Lanzhou, Gansu 730046, People's Republic of China

Full list of author information is available at the end of the article
}

the populations of Culicoides and the BTV replication cycle were both favored by the warm climate. Since 2006, BTV has spread extensively into several unexpected areas including Southern and Northern Europe, resulting in a serious economic burden [4-7].

A complex non-enveloped virus, BTV has a genome consisting of 10 segments of double-stranded RNA (dsRNA) encoding five different non-structural proteins, NS1, NS2, NS3, NS3A and NS4, as well as seven structural proteins (VP1-7) [8-11]. A BTV particle consists of three successive protein layers which form two capsids. The exterior capsid contains two major structural

(c) The Author(s). 2019 Open Access This article is distributed under the terms of the Creative Commons Attribution 4.0 International License (http://creativecommons.org/licenses/by/4.0/), which permits unrestricted use, distribution, and 
proteins, VP5 and VP2, while the interior capsid contains another two proteins, VP3 and VP7, and encloses a viral transcription complex composed of VP1 (polymerase), VP4 (capping enzyme), and VP6 (helicase) proteins, as well as the viral genome $[8,10,12,13]$. The non-structural proteins are mainly involved in virus assembly, replication, trafficking, release and morphogenesis $[9-11,14]$.

The transcriptome is a whole set of gene transcripts of specific cells, tissues, organs, or complete organisms, which associates the genetic information of the genome and the biological function of the proteome. The interaction between hosts or mammalian cells and pathogens such as Marek's disease virus, influenza virus, avian leukosis virus subgroups, bovine viral diarrhea virus, avian infectious bronchitis virus, Schmallenberg virus and tick-borne flaviviruses has been studied previously by transcriptome analysis [15-21]. Recently, deep sequencing has been considered to be a potent approach to transcriptome analyses which is superior to conventional methods in terms of repeatability and the falsepositive rate, as well as the dynamic scale [22, 23]. In this study, we used Aedes albopictus cells to reveal the transcriptome changes after infection with BTV, given the lack of the Culicoides genome sequence. Following this, several mRNA transcripts were selected to confirm the sequencing data by quantitative reverse transcription-polymerase chain reaction (qRT-PCR). The global transcriptome profiling will provide a deep understanding of BTV pathogenesis and virus-vector interactions.

\section{Methods}

\section{Cells and virus}

A. albopictus cells (ATCC-CCL-126) and BHK-21 cells (ATCC-CCL-10) were used in this study. A. albopictus cells were cultured in Dulbecco's modified Eagle's medium (DMEM, HyClone, USA) with the addition of $10 \%$ fetal bovine serum (FBS) (Gibco, USA) at a temperature of $28^{\circ} \mathrm{C}$. BHK-21 cells were cultured in modified Eagle's medium (MEM, HyClone) with the addition of $10 \% \mathrm{FBS}$ at $37^{\circ} \mathrm{C}$ with an atmosphere containing $5 \% \mathrm{CO}_{2}$. A BTV-1 strain (GS/11), which was isolated from sheep in western China in 1997, was propagated in BHK-21 cells and was used for viral infection. A BHK-21 monolayer was utilized to determine the virus titer, using the plaque formation assay [13].

\section{Immunofluorescence}

A. albopictus cells were seeded in 12-well plates and grown on glass coverslips (NEXT, China) and subsequently infected with BTV (multiplicity of infection $(\mathrm{MOI}=1))$ and incubated for 12 or $24 \mathrm{~h}$. Cells were then fixed with $4 \%$ paraformaldehyde (Solarbio, China), and permeabilized with $0.05 \%$ Triton X-100. BTV-infected fixed cells were incubated with a rabbit polyclonal antibody (1:1000) against recombinant BTV NS1 protein expressed in Escherichia coli (E.coli) and, subsequently, with the Alexa Fluor 568 anti-rabbit secondary antibody (Abcam, UK) (1:3000). Nuclei were counterstained with Hoechst 33258 (Invitrogen, USA). Cover glasses were mounted on glass slides using fluorescence mounting medium (ZSGB, China). Images were obtained using a fluorescence microscope (Leica, Germany).

\section{Western blot}

BTV-1 infection in A. albopictus cells was also confirmed by western blot analysis. BTV-infected and mock-infected cells in 12-well plates were harvested at 12 and $24 \mathrm{~h}$ post infection (hpi) with a cell scraper, separated at $1000 \times g$ in a centrifuge (Eppendorf $5424 \mathrm{R}$, Germany) for $5 \mathrm{~min}$, and the cell lysate pellet washed three times with phosphate-buffered saline (PBS). Cell lysates were denatured in $1 \times$ protein loading buffer (10 $\mathrm{mM}$ Tris- $\mathrm{HCl}, \mathrm{pH} 8.5,50 \mathrm{mM}$ DTT, $1 \%$ SDS, $10 \%$ glycerol, and $0.008 \%$ bromophenol blue) by heating for 5 min at $100^{\circ} \mathrm{C}$. SDS-PAGE was utilized to separate the proteins in the cell lysates, which were subsequently transferred onto nitrocellulose membranes (Millipore, USA). A blocking solution ( $0.5 \%$ Tween-20 and 5\% skimmed milk) was utilized to block the membranes for 1 h. Rabbit polyclonal antibodies against recombinant BTV1 NS1, NS2, and VP6 proteins expressed in E.coli in our laboratory, were used for probing, after which membranes were incubated with goat anti-rabbit IgG H\&L (alkaline phosphatase) secondary antibody (Abcam, UK).

\section{Virus infection}

To avoid contamination of BHK-21 cell debris, the virus used in this study was passaged three times in A. albopictus cells and then centrifuged after three freeze-thaws to remove the cell lysates. The supernatant was used to measure virus titers and to infect $A$. albopictus cells. A. albopictus cells were infected with BTV as described previously [24]. Briefly, to characterize the transcriptome profiles of $A$. albopictus cells after infection with BTV, $3 \times 10^{6}$ cells in $25-\mathrm{cm}^{2}$ flasks (Corning, USA) were infected in three replicates with a MOI of 1 . The cells were adsorbed with virus for $1 \mathrm{~h}$ at room temperature and then cultured in DMEM with the addition of $2 \%$ FBS. A albopictus cells without virus infection were used as the mock-infected group, in three replicates.

\section{RNA extraction and transcriptome sequencing}

BTV-infected and -mock-infected cells were collected at $24 \mathrm{hpi}$ with a cell scraper, separated at $1000 \times g$ in a centrifuge (Eppendorf $5810 \mathrm{R}$, Germany) for $5 \mathrm{~min}$, and the pellet washed three times in ice-cold PBS. Total RNA was extracted using a mixture of three replicated samples of 
cells using TRIzol reagent (Invitrogen, USA) and then digested with DNase I enzyme (TaKaRa, Japan). Oligo (dT) magnetic beads were utilized for poly (A) mRNA isolation, which was subsequently digested into fragments as a template for synthesizing first- strand cDNA using random primers and reverse transcriptase. RNase H, dNTPs, and DNA polymerase I were used to synthesize secondstrand $\mathrm{CDNA}$, which was then purified, repaired at the ends, connected with sequencing adaptors, and amplified by PCR to create a cDNA library using the Truseq ${ }^{\mathrm{qm}} \mathrm{RNA}$ Sample Prep Kit (Illumina, USA). The library was evaluated with an Agilent 2100 Bioanalyzer (Agilent Technologies, USA) and StepOne Plus Real-time PCR System (Applied Biosystems, USA), and was sequenced using an Illumina HiSeq ${ }^{\text {mi }} 2000$ sequencer (Illumina). The aforementioned RNA samples were utilized for qRT-PCR analysis of selected mRNA transcripts.

\section{Deep sequencing analyses}

Firstly, empty reads and adaptors, as well as reads filtered for low quality, were removed. Secondly, the reads were mapped to the $A$. aegypti genome using Bowtie software [25]. Lombardo et al. reported that several of the transcripts identified in A. albopictus showed a good level (70-100\%) of similarity with their A. aegypti homologs [26]. RNASeq by Expectation Maximization (RSEM) software (http://deweylab.biostat.wisc.edu/rsem/) was utilized to analyze differentially expressed genes and to quantify transcripts [27]. The filtering standard for the data was a false-discovery rate-corrected $P$ value $(q$ value) $<0.001$ and a fold- change $>2$. The databases local BLAST, Cluster of Orthologous Groups (COG), STRING and SwissProt were applied to predict and annotate all unigenes. The unigenes were analyzed by the Blast2GO tool on the basis of Gene Ontology (GO) terms. The mRNAs exhibiting differential expression were entered into the databases UniProt and the integrated discovery (DAVID) online server (http://david.abcc.ncifcrf.gov) to be annotated and visualized. The analyses included classifications of cell constituents and molecular function, as well as biological processes, with a confidence level of 95\%. The mRNA transcripts identified were grouped and classified by Kyoto Encyclopedia of Genes and Genomes (KEGG) pathway analysis. The STRING 10 database (http://string.embl.de/) was utilized to analyze the network of interactions between proteins, based on the identified mRNA transcripts [28].

\section{Validation of sequencing data}

qRT-PCR was used for the detection of several selected mRNAs with differential expression, with the aim of confirming the data of RNA-Seq. The afore-described protocol was used to prepare total RNA, which was subsequently digested using DNaseI enzyme (Promega,
USA). The Mx3500p system (Agilent Technologies) was applied to perform qRT-PCR. First-strand cDNAs were synthesized using the PrimerScript RT Master Mix (TaKaRa, Japan). A SYBR Premix Ex Taq ${ }^{\text {tax }}$ kit (TaKaRa) was used to perform real-time PCR, following the manufacturer's instructions. The PCR program was set as: $95^{\circ} \mathrm{C}$ for $30 \mathrm{~s}$, then 38 cycles of $95^{\circ} \mathrm{C}$ for $5 \mathrm{~s}$ and $60^{\circ} \mathrm{C}$ for $20 \mathrm{~s}$. Table 1 shows the respective primer sequences of the reference gene $\beta$-actin (A. albopictus) and selected mRNA transcripts. Each assay was conducted in three replicates. The method of $2^{-\Delta \Delta \mathrm{CT}}$ was used to calculate the relative expression levels of mRNAs in cells after BTV infection, which were expressed as the relative fold-change in the expression level in infected cells divided by that in control cells [29].

\section{Results}

\section{BTV infection in A. albopictus cells}

To examine the transcriptomic responses, BTV-1 was used to infect $A$. albopictus cells with a MOI of 1 to ensure a high ratio of virion to cells. As described previously, no morphological changes occurred in $A$. albopictus cells infected with BTV, which led to nonlytic infection without cytopathic effect (CPE) [24]. To confirm viral replication in A. albopictus cells following BTV infection, immunofluorescence, and western blot

Table 1 Primer sequences for analysis of gene expression using qRT-PCR

\begin{tabular}{lll}
\hline Primer name & Sequence (5'-3') & Product size (bp) \\
\hline AAEL015390-F & AGGCGAAGCCAAAGCAGTT & 90 \\
AAEL015390-R & TCGTGCGGTCTTCAGGTGT & \\
AAEL010126-F & TCCACCTCGTCGTCACCTTG & 131 \\
AAEL010126-R & TGTCGTCGTAGCTGTCGCT & \\
AAEL009532-F & CTGCTGTTCCACACGCTGAC & 189 \\
AAEL009532-R & TAACCGCGCTCTCCGAATGT & \\
AAEL001603-F & GCTGCCCATCCAGAACAAGC & 163 \\
AAEL001603-R & GGTGCGGCCACTGTATGTG & \\
AAEL012071-F & GCGCGTCAAAGATGCAGAGG & 166 \\
AAEL012071-R & GAAGGCATCGTCGACTCCCA & \\
AAEL001165-F & ACAGTTCGGCCAACTCGTCA & 149 \\
AAEL001165-R & ACTGGCTGTGGTGACTGCT & \\
AAEL002903-F & TTGCGCACCATCCAAGACG & 139 \\
AAEL002903-R & GTGCCCAATGTGCTGGTGT \\
AAEL004715-F & CGACGGGTAGCAGTAGCTGT & 150 \\
AAEL004715-R & TGTGGGCTTGCTCTGCGTT \\
AAEL010488-F & CCGTCTCCCAGTCACCTGTC \\
AAEL010488-R & CGTCATCCTGTGGCTGTGC \\
$\beta-$ - actin-F & GGAGAAGATCTGGCATCACA & 95 \\
$\beta$ - aCtin-R & TGTCATCTTCTCGCGGTTAG & \\
\hline
\end{tabular}


assays were performed using antibodies against NS1, NS2, and VP6 proteins. The immunofluorescence assay showed that NS1 was found in the cytoplasm and almost all cells were positive for BTV NS1 expression at 12 and 24 hpi (Fig. 1a). The NS1, NS2, and VP6 proteins were also detected at 12 and 24 hpi by western blot (Fig. 1b). These results confirmed BTV replication in A. albopictus cells, although no CPE appeared.

\section{Transcriptome alteration in A. albopictus cells infected with BTV}

To identify host cell transcripts involved in BTV-1 infection, libraries of mRNAs from BTV-infected and -mockinfected A. albopictus cells at $24 \mathrm{hpi}$ were submitted to high-throughput sequencing. In total, 51,850,205 raw reads were generated from the BTV infection group and $51,852,293$ from the control group. After empty reads and adapter sequences, as well as sequences of poor quality, were removed, 49,983,598 clean reads were obtained from the infection group and 49,762,646 from the control group (Fig. 2). In total, 12,822,376 clean reads in the infection group and 14,240,610 in the control group were completely mapped to the $A$. aegypti genome by Bowtie software. The majority of unigenes (5769; accounting for $80.6 \%$ of the total unigenes) were represented in both infection and control groups. However, 779 unigenes, which accounted for $10.9 \%$ of the total unigenes, existed exclusively in the infection group, while 607 unigenes, which accounted for $8.5 \%$ of the total unigenes, existed exclusively in the control group (Additional file 1). Differential expression analyses were performed using RSEM, and genes with a fold-change value $\geq 2$ ( $\mid \log _{2}$ fold change $\mid \geq 1$ ) and $q$ values $<0.001$ were accepted as significant. A total of 380 differentially expressed genes were detected, which indicated that the genes were related to BTV infection (Fig. 3, Additional file 2). Among the mRNA transcripts with differential expression, the expression of 362 genes was up-regulated, with fold differences ranging from 15.9- to 1-fold threshold value, and 18 genes were down-regulated, with fold differences ranging from - 12.8- to - 1-fold threshold value (Additional file 2).

\section{Bioinformatics analyses}

To further examine the biological functions of the 380 differentially expressed genes in A. albopictus cells upon BTV infection, GO terms were used to classify the functions of the BTV-infected cell transcripts, producing 603 terms for biological processes, 98 for cellular components, and 129 for molecular functions (Additional file 3). The annotation of biological processes indicated that most of the proteins under differential regulation were related to cellular and developmental processes, localization, biological regulation, metabolic processes and response to stimulus. It was shown by the annotation of cell constituents that most of the proteins with differential expression profiles were uniformly distributed in a variety of cellular constituents, including membranes, organelles and macromolecular complexes. It was revealed by the molecular

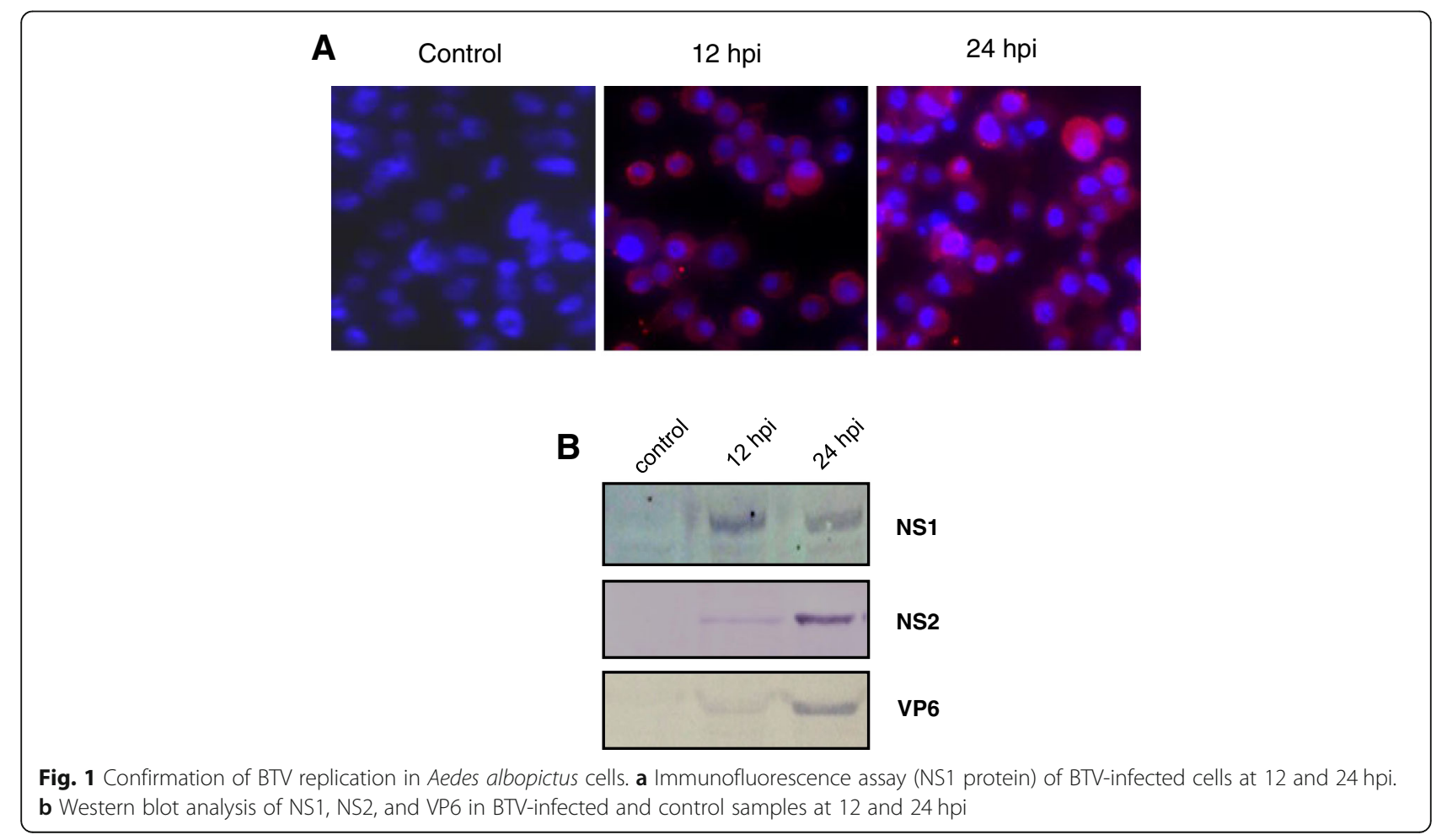


Classification of Raw Reads (Infected)

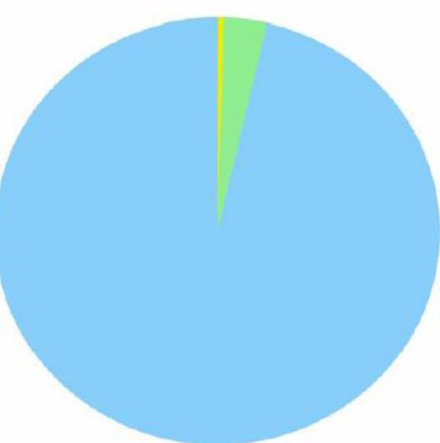

A
Classification of Raw Reads (Uninfected)

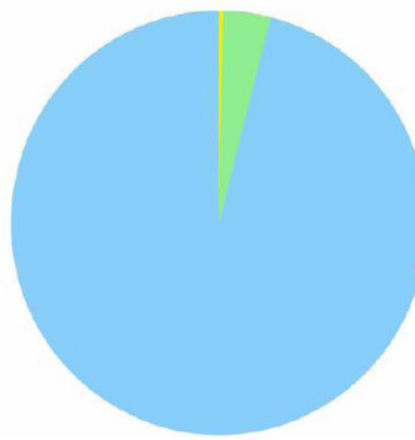

B

Fig. 2 Classification of raw reads from BTV-infected (a) and uninfected (b) Aedes albopictus cells

\section{Gene Expression Level (Uninfected-VS-Infected)}

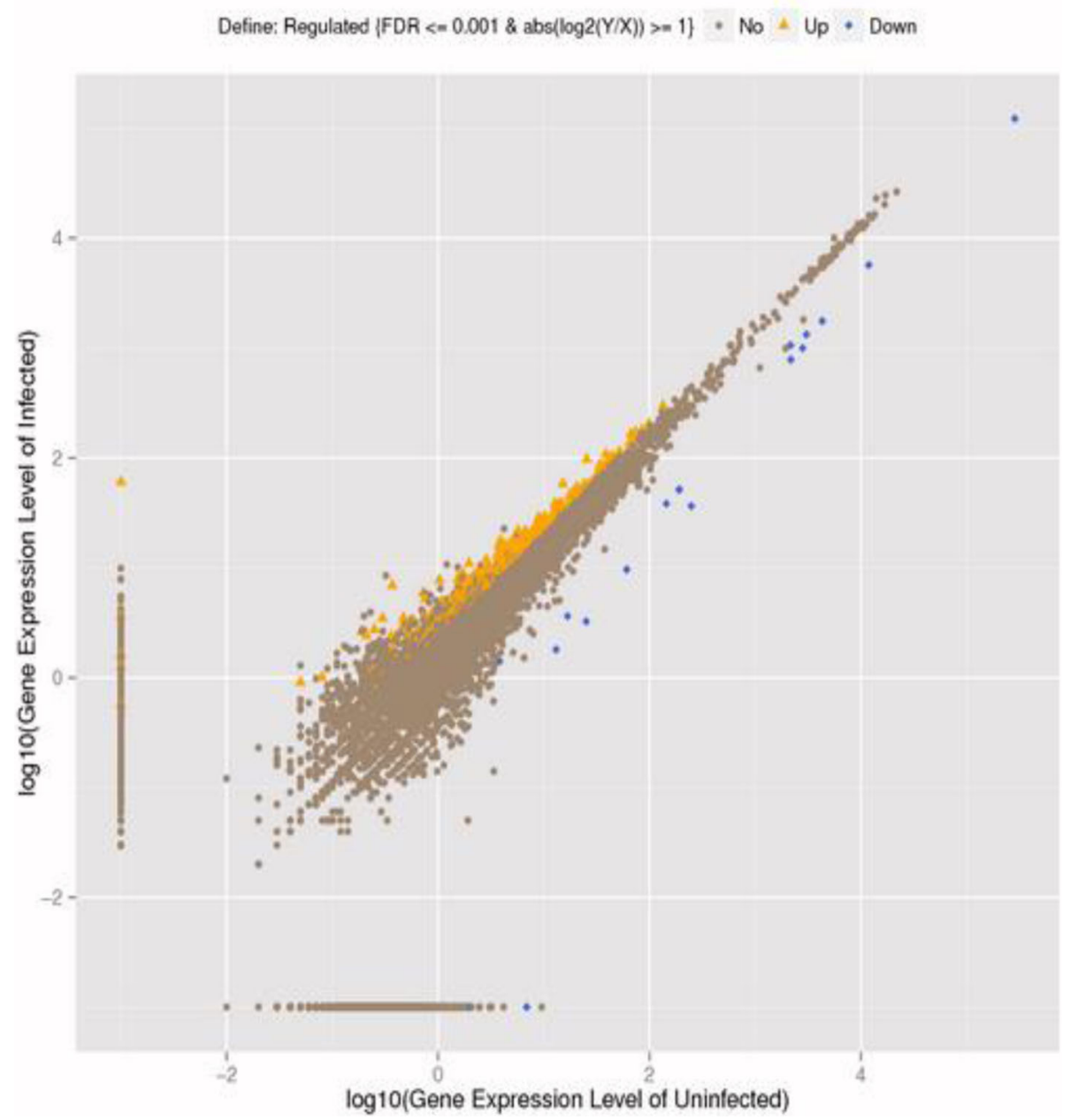

Fig. 3 Statistical charts of all expressed genes in BTV-infected and uninfected groups. The $x$ and $y$ axes represent the levels of expression of the mRNAs of the two groups. The yellow triangles represent mRNAs up-regulated in BTV-infected cells, and the blue points represent mRNAs downregulated in BTV-infected cells 
function annotation that virus-infected cells most frequently had changes in functions relating to binding and catalytic activity, as well as transporter function (Fig. 4). The KEGG pathway analyses showed that the genes with differential expression participated principally in endocytosis, FoxO, MAPK, dorso-ventral axis formation, insulin resistance, JAK-STAT, and Hippo signaling pathways (Additional file 4).

To clarify protein-protein interaction networks, the STRING database was searched to analyze further the 380 genes with differential expression. As shown in Fig. 5, several groups of proteins with strong interactions under significant regulation by BTV were detected, including AEL012071-AAEL002471-AAEL003845-AAEL00 0093-AAEL002594-AAEL004040 and AAEL010210-AAE L012421-AAEL010488-AAEL002663-AAEL005082-AAEL 005513. The above proteins have an essential role in innate immunity. The influence of BTV on the physiological function of infected cells was further clarified by analysis of interactive connections under potential regulation by BTV.

\section{Validation of differentially expressed mRNAs}

Transcriptome sequencing yields a large amount of data, and it is important to validate differential expression by independent methods. In order to confirm the results of the transcriptome sequencing, genes with differential expression were detected using qRT-PCR. Nine genes (AAEL015390, AAEL010126, AAEL009532, AAEL001603, AAEL012071, AAEL001165, AAEL002903, AAEL004715 and AAEL010488), which exhibited significant alterations in expression profiles after BTV infection, were validated using qRT-PCR. The result revealed that the relative expression levels of mRNAs of AAEL015390, AAEL010126, AAEL009532, AAEL001603, AAEL012071, AAEL001165, AAEL002903, AAEL004715 and AAEL010488 increased 3.66-, 3.09-, 4.26-, 3.56-, 1.89-, 2.61-, 2.42-, 2.78-, and 2.72-fold, respectively, in BTV-infected A. albopictus cells

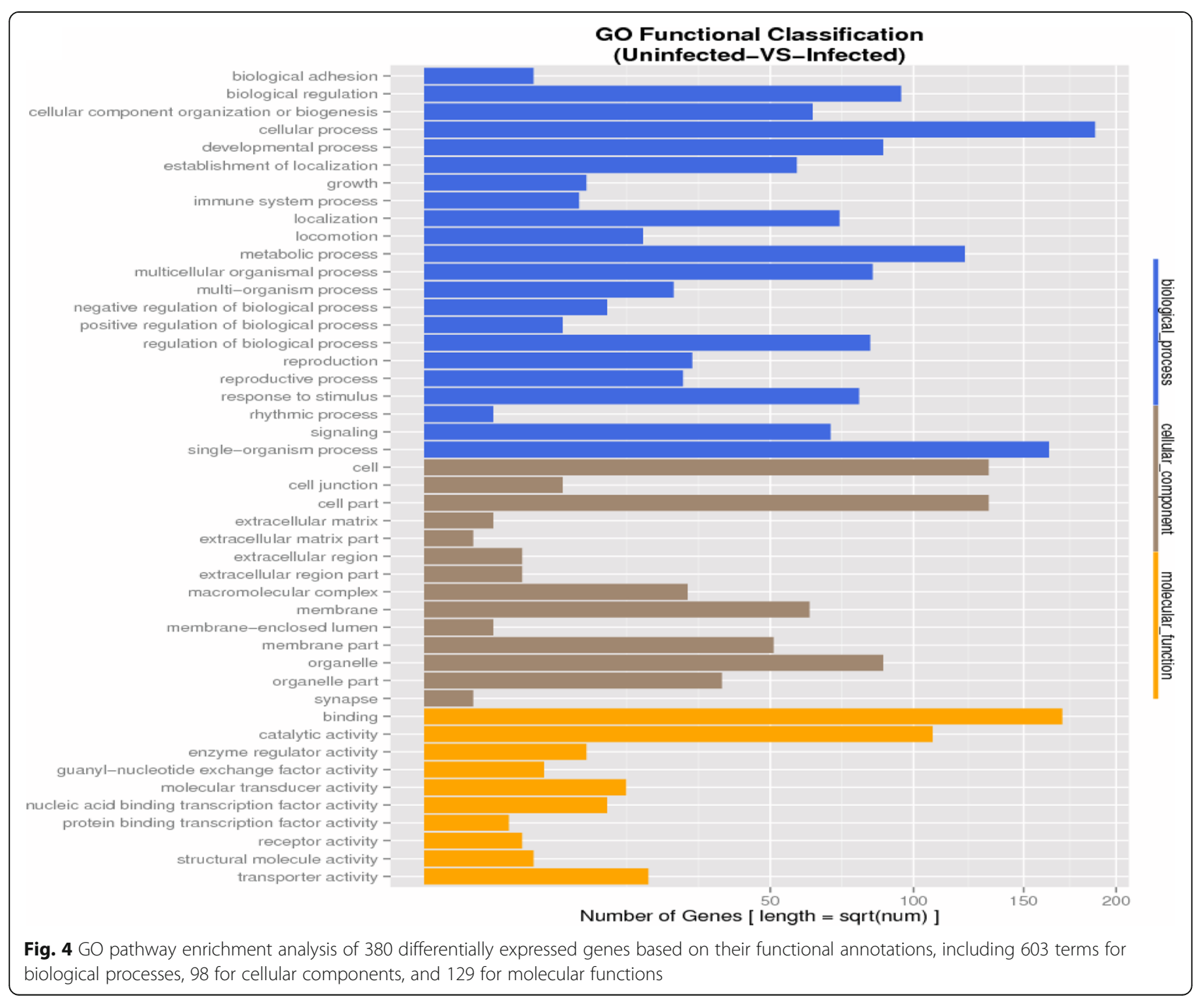




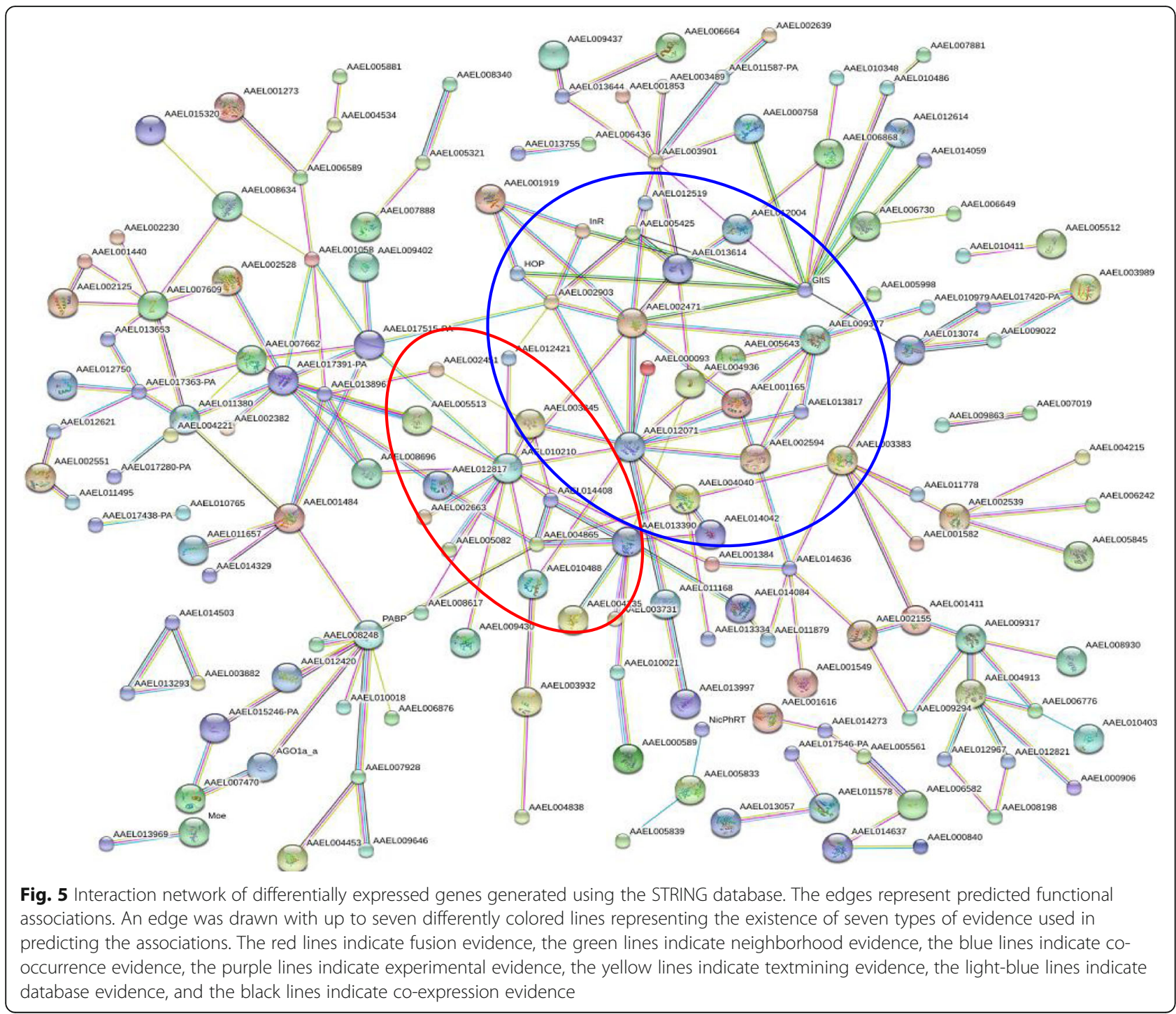

compared with the mock-infected cells at 24 hpi (Fig. 6), which conformed to the results of transcriptome sequencing, although there were fold differences between the qRT-PCR and transcriptome data, indicating that the expression profiles of differentially expressed genes in $A$. albopictus cells were notably changed in response to BTV infection.

\section{Discussion}

The infection of final hosts caused by arboviruses usually exhibits acute and pathogenic properties, while that in vectors is relatively moderate and non-pathogenic [3, 30]. It is well known that biting midges of the Culicoides genus are able to carry and transmit arboviruses, some of which result in infection among animals throughout the world, for example African horse sickness virus and BTV, as well as Schmallenberg virus, which was discovered recently $[3,7,31,32]$. BTV can induce a strong CPE in mammalian cells after infection while Culicoides- or mosquito-derived cell cultures cause non-lytic infection without obvious CPE [24, 33-36]. The study of interactions between arboviruses and Culicoides vectors has been restricted by the absence of Culicoides genome sequences $[37,38]$. A albopictus cells are derived from mosquitos and are usually used for studies of BTV and other arboviruses [39-42]. Nevertheless, data on alteration of the transcriptome of A. albopictus cells in response to BTV infection were previously unavailable. In this study, the transcriptome was sequenced for the identification of the mRNA expression pattern in BTV-infected A. albopictus cells. In total, $12,822,376$ and 14,240,610 clean reads were obtained from cells with and without BTV infection, respectively. A total of 380 differentially expressed genes (362 up-regulated and 18 down-regulated) were identified in the study, which strongly indicated that 


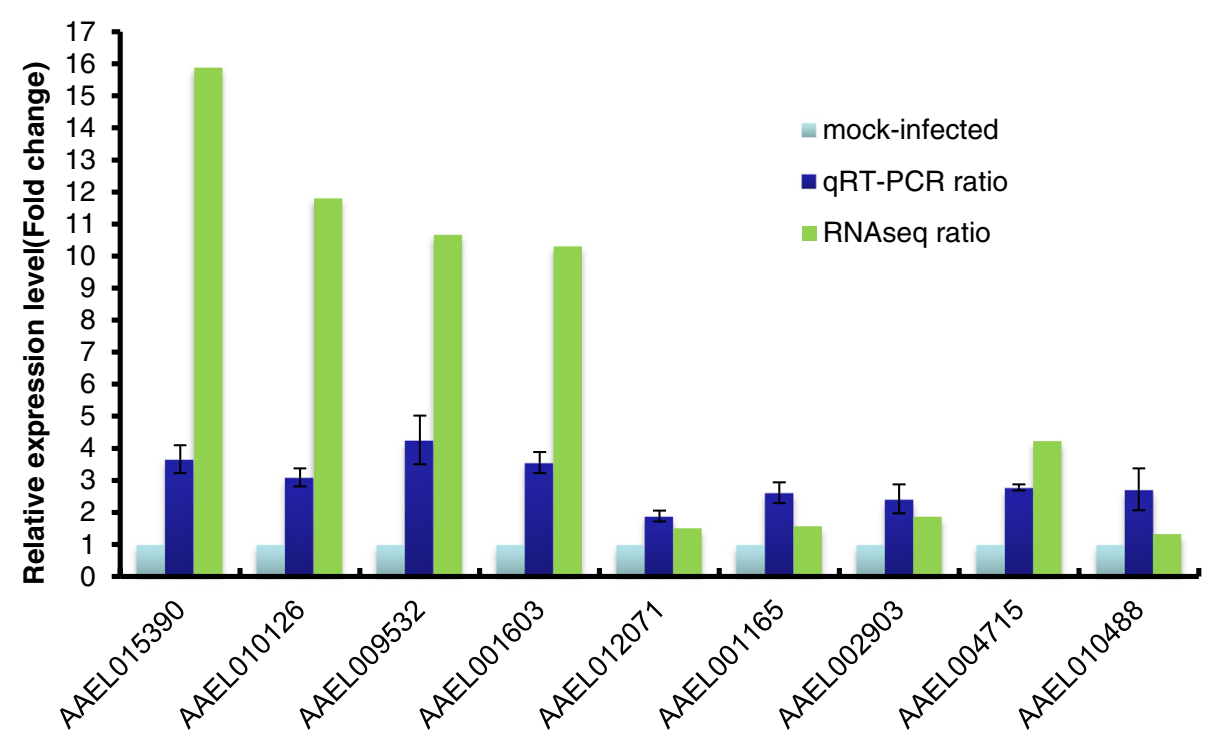

Fig. 6 qRT-PCR validation of nine selected mRNAs. The relative expression level of each mRNA transcript in BTV-infected cells was calculated using the $2^{-\Delta \Delta C T}$ method and represented as the $n$-fold change relative to the uninfected cells

the differentially expressed genes are involved in BTV infection. Production of mature viral particles was exponential at 8 and $24 \mathrm{hpi}$ [1]. With antiviral response in mind, we focused on transcriptome changes during the early stage of infection (24 hpi), which avoided RNA degradation and interference with cell maintenance at the late stage of infection in order to guarantee the quality of cDNA libraries for transcriptome sequencing. While our manuscript was in preparation, the genome of Culicoides sonorensis, a vector of BTV, was sequenced, which will facilitate the identification of potential antiviral factors and unravel the transmission mechanism of BTV as well as other arboviruses [43]. Soon, we will investigate the alterations in the transcriptome of Culicoides sonorensis $(\mathrm{KC})$ cells infected with BTV to see how the changes induced by $\mathrm{BTV}$ infection of $\mathrm{KC}$ cells differ from those in $A$. albopictus cells.

In insects, the infectious outcomes are notably influenced by the interactions between viruses and the innate immunity of the vectors, in spite of the evidence that the immune response of insects is similar to adaptive immunity in mammals $[38,44]$. One of the principal mechanisms of defense against viruses is RNA interference (RNAi), which inhibits viral replication by detecting dsRNA derived from viruses $[44,45]$. We recently performed deep sequencing to identify micro RNAs (miRNAs) with differential expression in BTV-infected $A$. albopictus cells. The results showed that 140 miRNAs with differential expression, including 125 novel candidates and 15 known miRNAs, were detected and predicted to be essential regulatory miRNAs in the early stage of BTV infection [24]. In addition to RNAi, other innate antiviral pathways such as Toll and JAK-STAT were also revealed as essential regulators of insect antiviral responses [44, 46-48]. Viruses, fungi, and Grampositive bacteria are principal activators of the Toll pathway, which, to a great extent, controls antimicrobial peptide (AMP) expression [49]. The JAK-STAT pathway was originally identified in mammals, and proved to play an essential role during infection by viruses such as dengue virus and Drosophila $C$ virus [50, 51]. Moreover, this pathway was found to be conserved in defense against viruses among insects and human beings [52, 53]. In this study, the JAK-STAT signaling pathway was also identified among the differentially expressed genes, but the Toll signaling pathway was not identified, indicating that the JAK-STAT and Toll pathways act in two distinct antiviral networks. This result is consistent with a recent report from our laboratory on miRNA expression analysis in BTV-infected A. albopictus cells [24]. These findings strongly indicate that the JAK-STAT pathway may have an important action in BTV-vector interaction.

Endocytosis provides pathways through which many viruses productively infect their target cells [54]. Different mechanisms are available for the endocytic internalization of BTV particles, including clathrin-mediated endocytosis and macropinocytosis [13, 55-57]. Herein, the results showed that several of the differentially expressed genes may be involved in endocytosis, which provides a possible insight into the pathway of BTV entry into A. albopictus cells. From our current data, it is not clear so far how this possible role of the differentially expressed genes would favor the overall infection 
of the A. albopictus cells by endocytic pathway. One possibility inviting speculation in this point is that the fraction of cells that are actually infected by BTV (note that our use of MOI of 1 , does not warrant infection of the totality of the cells) would somehow be upregulating the endocytic pathways in the yet-to-be-infected cells. BTV is capable of infecting a variety of cells, including HeLa, BHK-21, MDBK, KC and A. albopictus cells, and probably infects host mammalian cells and vectors through various mechanisms [13, 34, 55, 57]. The exact mechanism by which BTV infects $A$. albopictus cells requires further investigation.

Programmed cell death (PCD), also known as apoptosis, acts as an intrinsic response to viral infection, which is able to limit viral replication and growth in mammalian cells $[58,59]$. The replication of some arboviruses were also shown to be suppressed in a PCD manner in insect cells after infection [60-62]. Our results would suggest that the Hippo and FoxO signaling pathways, which are known to participate in PCD, were identified as differentially expressed in A. albopictus cells, which is consistent with our previous report [24]. These results are really intriguing at this time and future efforts need to be directed towards ascertaining whether Hippo and FoxO signaling pathways actively inhibit BTV replication in A. albopictus cells.

\section{Conclusion}

In summary, we investigated the alteration in the expression of mRNA transcripts with differential expression in BTV-infected $A$. albopictus cells by transcriptome sequencing. A total of 380 differentially expressed genes were detected, 362 of which were up-regulated and 18 of which were down-regulated. Bioinformatics analyses showed that the differentially expressed mRNAs were mainly involved in endocytosis, FoxO, MAPK, dorsoventral axis formation, insulin resistance, and the Hippo and JAK-STAT signaling pathways. Consequently, these differentially expressed mRNAs probably play an essential role in antiviral immune responses and viral pathogenesis in insects and insect cells. The results of this study may be helpful in identifying potential antiviral factors and providing molecular clues for unraveling the mechanism of non-lytic BTV infection, and that involving other arboviruses.

\section{Additional files}

Additional file 1: List of unigenes identified in BTV-infected and uninfected cells. (XLSX $1431 \mathrm{~kb}$ )

Additional file 2: List of differentially expressed genes. (XLSX 99 kb)
Additional file 3: GO annotations for differentially expressed genes. (XLS 394 kb)

Additional file 4: KEGG Pathway annotations for differentially expressed genes. (XLS $25 \mathrm{~kb}$ )

\section{Abbreviations}

AMP: Antimicrobial peptides; BT: Bluetongue; BTV: Bluetongue virus; CPE: Cytopathic effect; DAVID: Databases UniProt and the integrated discovery; DMEM: Dulbecco's modified Eagle's medium; dsRNA: Doublestranded RNA; FBS: Fetal bovine serum; GO: Gene Ontology; hpi: Hours post infection; KC: Culicoides sonorensis; KEGG: Kyoto Encyclopedia of Genes and Genomes; MEM: Modified Eagle's medium; MOI: Multiplicity of infection; PBS: Phosphate-buffered saline; PCD: Programmed cell death; PCR: Polymerase chain reaction; PFU: Plaque formation units; qRTPCR: Quantitative reverse transcription-polymerase chain reaction; RSEM: RNASeq by expectation maximization

\section{Acknowledgements}

We thank Dr. Antoinette Van Schalkwyk from Onderstepoort Veterinary Institute (South Africa) for critically reading the manuscript.

\section{Authors' contributions}

Experiments were conceived and designed by JD and HY. Experiments were performed by JD, YG, DK, SX and GZ, and the data were analyzed by JD, SG and ZT. The manuscript was written by JD and HY. GL, JL and HC helped to design the experiments and draft the manuscript. All authors read and approved the final manuscript.

\section{Funding}

This study was financially supported by the National Key Research and Development Program of China (2017YFD0502304); National Natural Science Foundation of China (31672562); China-South Africa Joint Research Project (CS08-L13); NBCIS (CARS-37); ASTIP(CAAS-ASTIP-2016-LVRI); Jiangsu Co-innovation Center Program for the Prevention and Control of Important Animal Infectious Diseases and Zoonoses. The funders had no role in the study design, data collection, analysis, and interpretation of data, and in writing the manuscript or decision to publish.

\section{Availability of data and materials}

All sequencing data generated in this study have been deposited at the Sequence Read Archive (SRA) database at National Center for Biotechnology Information (NCBI) under the accession number PRJNA436549.

Ethics approval and consent to participate

Not applicable

Consent for publication

Not applicable

\section{Competing interests}

The authors declare that they have no competing interests.

\section{Author details}

${ }^{1}$ State Key Laboratory of Veterinary Etiological Biology, Lanzhou Veterinary Research Institute, Chinese Academy of Agricultural Sciences, Xujiaping 1, Lanzhou, Gansu 730046, People's Republic of China. ${ }^{2}$ Jiangsu Co-innovation Center for Prevention and Control of Important Animal Infectious Diseases and Zoonoses, Yangzhou University, Yangzhou 225009, People's Republic of China.

Received: 20 April 2018 Accepted: 31 May 2019

Published online: 10 June 2019

References

1. Schwartz-Cornil I, Mertens PP, Contreras V, Hemati B, Pascale F, Breard E, Mellor PS, MacLachlan NJ, Zientara S. Bluetongue virus: virology, pathogenesis and immunity. Vet Res. 2008;39(5):46.

2. MacLachlan NJ. The changing global face of bluetongue: from the beginning. Vet Ital. 2015;51(4):249-51. 
3. Mellor PS, Boorman J, Baylis M. Culicoides biting midges: their role as arbovirus vectors. Annu Rev Entomol. 2000;45:307-40.

4. Enserink M. Emerging infectious diseases. During a hot summer, bluetongue virus invades northern Europe. Science. 2006;313(5791):1218-9.

5. Zientara S, Sanchez-Vizcaino JM. Control of bluetongue in Europe. Vet Microbiol. 2013;165(1-2):33-7.

6. Toussaint JF, Vandenbussche F, Mast J, De Meester L, Goris N, Van Dessel W, Vanopdenbosche E, Kerkhofs P, De Clercq K, Zientara S, et al. Bluetongue in northern Europe. Vet Rec. 2006;159(10):327.

7. Mellor PS, Carpenter S, Harrup L, Baylis M, Mertens PP. Bluetongue in Europe and the Mediterranean Basin: history of occurrence prior to 2006. Prev Vet Med. 2008:87(1-2):4-20.

8. Patel A, Roy P. The molecular biology of bluetongue virus replication. Virus Res. 2014;182:5-20.

9. Roy P. Bluetongue virus: dissection of the polymerase complex. J Gen Virol. 2008:89(Pt 8):1789-804.

10. Roy P. Functional mapping of bluetongue virus proteins and their interactions with host proteins during virus replication. Cell Biochem Biophys. 2008;50(3):143-57.

11. Ratinier M, Shaw AE, Barry G, Gu Q, Di Gialleonardo L, Janowicz A, Varela M, Randall RE, Caporale M, Palmarini M. Bluetongue virus NS4 protein is an interferon antagonist and a determinant of virus virulence. J Virol. 2016; 90(11):5427-39.

12. Matsuo E, Roy P. Bluetongue virus VP6 acts early in the replication cycle and can form the basis of chimeric virus formation. J Virol. 2009;83(17):8842-8.

13. Du J, Bhattacharya B, Ward TH, Roy P. Trafficking of bluetongue virus visualized by recovery of tetracysteine-tagged virion particles. J Virol. 2014; 88(21):12656-68

14. Feenstra F, Drolet BS, Boonstra J, van Rijn PA. Non-structural protein NS3/ NS3a is required for propagation of bluetongue virus in Culicoides sonorensis. Parasit Vectors. 2015:8:476.

15. Villalba M, Fredericksen F, Otth C, Olavarria V. Transcriptomic analysis of responses to cytopathic bovine viral diarrhea virus-1 (BVDV-1) infection in MDBK cells. Mol Immunol. 2016;71:192-202.

16. Chen C, Li H, Xie Q, Shang H, Ji J, Bai S, Cao Y, Ma Y, Bi Y. Transcriptional profiling of host gene expression in chicken liver tissues infected with oncogenic Marek's disease virus. J Gen Virol. 2011;92(Pt 12:2724-33.

17. Li Y, Zhou H, Wen Z, Wu S, Huang C, Jia G, Chen H, Jin M. Transcription analysis on response of swine lung to $\mathrm{H} 1 \mathrm{~N} 1$ swine influenza virus. BMC Genomics. 2011;12:398

18. Hang B, Sang J, Qin A, Qian K, Shao H, Mei M, Ye J. Transcription analysis of the response of chicken bursa of Fabricius to avian leukosis virus subgroup J strain JS09GY3. Virus Res. 2014;188:8-14.

19. Blomstrom AL, Gu Q, Barry G, Wilkie G, Skelton JK, Baird M, McFarlane M, Schnettler E, Elliott RM, Palmarini M, et al. Transcriptome analysis reveals the host response to Schmallenberg virus in bovine cells and antagonistic effects of the NSs protein. BMC Genomics. 2015;16:324.

20. Mlera L, Lam J, Offerdahl DK, Martens C, Sturdevant D, Turner CV, Porcella SF, Bloom ME. Transcriptome Analysis Reveals a Signature Profile for TickBorne Flavivirus Persistence in HEK 293T Cells. MBio. 2016;7(3):e00314-16.

21. Cong F, Liu X, Han Z, Shao Y, Kong X, Liu S. Transcriptome analysis of chicken kidney tissues following coronavirus avian infectious bronchitis virus infection. BMC Genomics. 2013;14:743.

22. Tariq MA, Kim HJ, Jejelowo O, Pourmand N. Whole-transcriptome RNAseq analysis from minute amount of total RNA. Nucleic Acids Res. 2011;39(18): e120.

23. Sultan M, Schulz MH, Richard $H$, Magen A, Klingenhoff $A$, Scherf $M$, Seifert M, Borodina T, Soldatov A, Parkhomchuk D, et al. A global view of gene activity and alternative splicing by deep sequencing of the human transcriptome. Science. 2008:321(5891):956-60.

24. Xing S, Du J, Gao S, Tian Z, Zheng Y, Liu G, Luo J, Yin H. Analysis of the miRNA expression profile in an Aedes albopictus cell line in response to bluetongue virus infection. Infect Genet Evol. 2016;39:74-84.

25. Nene V, Wortman JR, Lawson D, Haas B, Kodira C, Tu ZJ, Loftus B, Xi Z, Megy K, Grabherr M, et al. Genome sequence of Aedes aegypti, a major arbovirus vector. Science. 2007;316(5832):1718-23.

26. Lombardo F, Salvemini M, Fiorillo C, Nolan T, Zwiebel L, Ribeiro JM, Arca B. Deciphering the olfactory repertoire of the tiger mosquito Aedes albopictus. BMC Genomics. 2017;18(1):770.

27. Li B, Dewey CN. RSEM: accurate transcript quantification from RNA-Seq data with or without a reference genome. BMC Bioinformatics. 2011;12:323.
28. Szklarczyk D, Morris JH, Cook H, Kuhn M, Wyder S, Simonovic M, Santos A, Doncheva NT, Roth A, Bork P, et al. The STRING database in 2017: qualitycontrolled protein-protein association networks, made broadly accessible. Nucleic Acids Res. 2017;45(D1):D362-8.

29. Schmittgen TD, Livak KJ. Analyzing real-time PCR data by the comparative C(T) method. Nat Protoc. 2008;3(6):1101-8.

30. Patterson J, Sammon M, Garg M. Dengue, Zika and chikungunya: emerging arboviruses in the New World. West J Emerg Med. 2016;17(6):671-9.

31. De Regge N, Deblauwe I, De Deken R, Vantieghem P, Madder M, Geysen D, Smeets F, Losson B, van den Berg T, Cay AB. Detection of Schmallenberg virus in different Culicoides spp. by real-time RT-PCR. Transbound Emerg Dis. 2012;59(6):471-5.

32. Mellor PS, Boorman J. The transmission and geographical spread of African horse sickness and bluetongue viruses. Ann Trop Med Parasitol. 1995;89(1): $1-15$.

33. van Gennip RG, van de Water SG, van Rijn PA. Bluetongue virus nonstructural protein NS3/NS3a is not essential for virus replication. PLoS One. 2014;9(1):e85788.

34. Wechsler SJ, McHolland LE. Susceptibilities of 14 cell lines to bluetongue virus infection. J Clin Microbiol. 1988;26(11):2324-7.

35. Ftaich N, Ciancia C, Viarouge C, Barry G, Ratinier M, van Rijn PA, Breard E, Vitour D, Zientara S, Palmarini M, et al. Turnover rate of NS3 proteins modulates bluetongue virus replication kinetics in a host-specific manner. J Virol. 2015;89(20):10467-81.

36. Celma CC, Roy P. A viral nonstructural protein regulates bluetongue virus trafficking and release. J Virol. 2009;83(13):6806-16.

37. Nayduch D, Cohnstaedt LW, Saski C, Lawson D, Kersey P, Fife M, Carpenter S. Studying Culicoides vectors of BTV in the post-genomic era: resources, bottlenecks to progress and future directions. Virus Res. 2014;182:43-9.

38. Fragkoudis R, Attarzadeh-Yazdi G, Nash AA, Fazakerley JK, Kohl A. Advances in dissecting mosquito innate immune responses to arbovirus infection. J Gen Virol. 2009;90(Pt 9:2061-72.

39. Offerdahl DK, Dorward DW, Hansen BT, Bloom ME. Cytoarchitecture of Zika virus infection in human neuroblastoma and Aedes albopictus cell lines. Virology. 2017;501:54-62.

40. Mertens PP, Burroughs JN, Walton A, Wellby MP, Fu H, O'Hara RS, Brookes SM, Mellor PS. Enhanced infectivity of modified bluetongue virus particles for two insect cell lines and for two Culicoides vector species. Virology. 1996;217(2):582-93.

41. Eschbaumer M, Wernike K, Batten CA, Savini G, Edwards L, Di Gennaro A, Teodori L, Oura CA, Beer M, Hoffmann B. Epizootic hemorrhagic disease virus serotype 7 in European cattle and sheep: diagnostic considerations and effect of previous BTV exposure. Vet Microbiol. 2012;159(3-4):298-306.

42. Li M, Zheng Y, Zhao G, Fu S, Wang D, Wang Z, Liang G. Tibet Orbivirus, a novel Orbivirus species isolated from Anopheles maculatus mosquitoes in Tibet, China. PLoS One. 2014;9(2):e88738.

43. Morales-Hojas R, Hinsley M, Armean IM, Silk R, Harrup LE, Gonzalez-Uriarte A, Veronesi $E$, Campbell L, Nayduch D, Saski C, et al. The genome of the biting midge Culicoides sonorensis and gene expression analyses of vector competence for bluetongue virus. BMC Genomics. 2018;19(1):624

44. Kingsolver MB, Huang Z, Hardy RW. Insect antiviral innate immunity: pathways, effectors, and connections. J Mol Biol. 2013;425(24):4921-36.

45. Myles KM, Wiley MR, Morazzani EM, Adelman ZN. Alphavirus-derived small RNAs modulate pathogenesis in disease vector mosquitoes. Proc Natl Acad Sci U S A. 2008;105(50):19938-43.

46. Xi Z, Ramirez JL, Dimopoulos G. The Aedes aegypti toll pathway controls dengue virus infection. PLoS Pathog. 2008;4(7):e1000098.

47. Lemaitre B, Hoffmann J. The host defense of Drosophila melanogaster. Annu Rev Immunol. 2007;25:697-743.

48. Fragkoudis R, Chi Y, Siu RW, Barry G, Attarzadeh-Yazdi G, Merits A, Nash AA, Fazakerley JK, Kohl A. Semliki Forest virus strongly reduces mosquito host defence signaling. Insect Mol Biol. 2008;17(6):647-56.

49. Tzou P, Reichhart JM, Lemaitre B. Constitutive expression of a single antimicrobial peptide can restore wild-type resistance to infection in immunodeficient Drosophila mutants. Proc Natl Acad Sci U S A. 2002;99(4): 2152-7.

50. Souza-Neto JA, Sim S, Dimopoulos G. An evolutionary conserved function of the JAK-STAT pathway in anti-dengue defense. Proc Natl Acad Sci U S A. 2009;106(42):17841-6.

51. Silvennoinen $\mathrm{O}$, Ihle JN, Schlessinger J, Levy DE. Interferon-induced nuclear signalling by Jak protein tyrosine kinases. Nature. 1993;366(6455):583-5. 
52. Arbouzova NI, Zeidler MP. JAK/STAT signalling in Drosophila: insights into conserved regulatory and cellular functions. Development. 2006;133(14): 2605-16.

53. Hedges LM, Johnson KN. Induction of host defence responses by Drosophila C virus. J Gen Virol. 2008;89(Pt 6:1497-501.

54. Marsh M, Helenius A. Virus entry: open sesame. Cell. 2006;124(4):729-40.

55. Forzan M, Marsh M, Roy P. Bluetongue virus entry into cells. J Virol. 2007 81(9):4819-27.

56. Patel A, Mohl BP, Roy P. Entry of bluetongue virus capsid requires the late endosome-specific lipid Lysobisphosphatidic acid. J Biol Chem. 2016;291(23): 12408-19.

57. Gold S, Monaghan P, Mertens P, Jackson T. A Clathrin Independent Macropinocytosis-Like Entry Mechanism Used by Bluetongue Virus-1 during Infection of BHK Cells. Plos One. 2010;5(6):e11360.

58. Upton JW, Chan FK. Staying alive: cell death in antiviral immunity. Mol Cell. 2014:54(2):273-80.

59. Lamkanfi M, Dixit VM. Manipulation of host cell death pathways during microbial infections. Cell Host Microbe. 2010;8(1):44-54.

60. Blair CD, Olson KE. Mosquito immune responses to arbovirus infections. Curr Opin Insect Sci. 2014;3:22-9.

61. Clarke TE, Clem RJ. Insect defenses against virus infection: the role of apoptosis. Int Rev Immunol. 2003;22(5-6):401-24.

62. O'Neill K, Olson BJ, Huang N, Unis D, Clem RJ. Rapid selection against arbovirus-induced apoptosis during infection of a mosquito vector. Proc Natl Acad Sci U S A. 2015;112(10):E1152-61.

\section{Publisher's Note}

Springer Nature remains neutral with regard to jurisdictional claims in published maps and institutional affiliations.

Ready to submit your research? Choose BMC and benefit from:

- fast, convenient online submission

- thorough peer review by experienced researchers in your field

- rapid publication on acceptance

- support for research data, including large and complex data types

- gold Open Access which fosters wider collaboration and increased citations

- maximum visibility for your research: over $100 \mathrm{M}$ website views per year

At BMC, research is always in progress.

Learn more biomedcentral.com/submissions 